\title{
LA PRIMERA CALLE, LA PRIMERA MARGINACIÓN: EL BULLYING EN UN ESPACIO MARGINAL DE LA CAPITAL DE CUBA
}

\author{
Cecilia Alonso Martínez \\ Casa de las Américas. Cuba
}

Recibido 30-04-2015

Aceptado 25-05-2015

\section{Resumen}

En el presente artículo se estudia la necesidad de la integración de los niños y adolescentes de El Fanguito y Las Canteras para evitar el bullying social y la discriminación de género. Aquí se analizan las afectaciones que imprime la pobreza a la búsqueda de soluciones creativas ante las necesidades materiales y se exponen las consecuencias que tiene vivir en un barrio marginal para la construcción de la imagen del mundo. Finalmente se explican algunas vías para eliminar las conductas negativas y construir un espacio de diálogo Fanguito-Canteras.

Palabras clave: integración, marginalidad, pobreza, río, diálogo, educación de adultos.

\begin{abstract}
This paper studies the need of integration for kids and adolescents of El Fanguito and Las Canteras to avoid social bullying and gender discrimination. It also analyzes the limitations imposed by poverty in order to search creative solutions for the material needs and it also exposes the consequences of living in a marginal place in the construction of the imago world. Finally, it exposes the solutions for negative manners and proposes a way for the construction of the dialogue Fanguito-Canteras.
\end{abstract}

Key-words: integration, marginalization, poverty, river, dialogue, adult education. 



\section{Introducción}

En los últimos veinte años, aproximadamente, la pobreza es un tema retomado en Cuba para las investigaciones de las ciencias sociales, ¿es que acaso desapareció entre 1959 y 1989? En realidad lo que ocurrió fue que «Se creó un escenario subjetivo de optimismo social compartido, que indujo a la idea de la total desaparición de la pobreza y de que insistir desde las ciencias sociales en dicha problemática podía parecer un acto crítico excesivo o de mala fe» ${ }^{1}$.

Sin embargo, la pobreza como fenómeno multidimensional no se suprimió. Aunque en los primeros treinta años de su revolución Cuba consiguió eliminar los fundamentos de la pobreza que tenían lugar en las relaciones de explotación y el desamparo, no pudo alterar su posición de país periférico y pobre en el contexto internacional, lo que continuó incidiendo en sus efectivos para la atención a las políticas de desarrollo social.

Por ello, cuando en los noventa surge la crisis y con ella la reforma,

No estaba configurado totalmente y no podían estar, por tanto, resueltos totalmente los problemas inherentes a la superación de desigualdades y desventajas sociales. Su composición social, incluso con anterioridad a la crisis y la reforma, aunque había avanzado considerablemente en materia de igualdad, era diversa y expresaba diferentes grados de acceso a las ventajas sociales y satisfacción de necesidades².

El sistema cubano procedió a reproducirse simbólica e ideológicamente en su reforma económica a través de instrumentos de inclusión social y cuidado a los derechos sociales. También se distinguió la reforma por la no mercantilización del empleo y por su permanencia en poder estatal. Es lógico que en estas circunstancias cambie la correspondencia Estado-mercado, «con claros efectos sobre los niveles de igualdad, los mecanismos de distribución y las políticas sociales» ${ }^{3}$.

1. EsPinA, Mayra Paula. «El caso cubano en diálogo de contraste». En Políticas de atención a la pobreza y la desigualdad. Examinando el rol del estado en la experiencia cubana. Buenos Aires, Consejo Latinoamericano de Ciencias Sociales (CLACSO), 2008, p. 98.

2. Ibíd., p. 112.

3. Ibíd., p. 136.

Feminismo/s 25, junio 2015, pp. 55-70 
En comparación con América Latina y el Caribe, las autoras Espina y Ferriol coinciden en que Cuba no ostenta el peor puesto de la región ${ }^{4}$; sino que posee condiciones muy propias que la doctora Espina denomina «situación general de pobreza amparada»,

Como regla, tendríamos en Cuba pobres integrados que gozan de servicios públicos de alta calidad, servicios que son exactamente los mismos a los que acceden todos los grupos de ingresos y que incluyen estudios universitarios, trasplantes de órganos, vacunas variadas, pero a los que les resulta muy difícil cubrir sus demandas de alimentos y otras necesidades básicas que se satisfacen en el hogar, en buena medida a través del mercado. A ello se suma la dificultad para disponer de una vivienda adecuada. Se trata de una situación en la que el ámbito de generación autónoma de medios propios para satisfacer necesidades y elegir sus satisfacciones está seriamente dañado ${ }^{5}$.

A pesar de estos niveles de amparo, existe, además, una situación actual de franjas de pobreza no cuantificada, y en algunos casos desprotegida de la universalidad garantizada por el Estado. Se trata de asentamientos marginales autoproducidos y espontáneos que no se benefician de un reconocimiento legal por ser improvisados y asociados a las carencias de vivienda dadas por la migración hacia territorios de mayores oportunidades económicas. Este circuito que Espina denomina como más crítico en cuestiones de pobreza es el más preocupante de la situación actual de Cuba, sería importante y necesaria la creación de políticas sociales en las que se pueda combinar la focalización con la universalización; retos a los que Cuba se ve enfrentada actualmente.

El presente estudio de caso El Fanguito-Las Canteras se encuentra dentro de este margen de pobreza en un espacio de la capital cubana en el municipio Plaza de la Revolución (El Vedado), ampliamente conocido por el confort en la vida de sus vecinos, mientras van creciendo a su alrededor estos bolsones de pobreza. El estudio realizado por el Dr. Alberto Dorta Contreras ${ }^{6}$ ha conseguido medir el Índice de Desarrollo Humano en los 15 municipios de la provincia La Habana, aplicando un cálculo de Índice de Desarrollo Humano Territorial obtuvo como dato más reciente que en el año 2010 el municipio

4. EsPina, Mayra Paula. Op. cit., p. 179; FerRIOL, Ángela. Políticas Sociales y reformas estructurales: Cuba a principios del siglo XXI. Comisión Económica para América Latina y el Caribe (CEPAL), Naciones Unidas, Programa de las Naciones Unidas para el Desarrollo (PNUD), Instituto Nacional de Investigaciones Económicas (INIE), 2004, p. 68.

5. EsPina, Mayra Paula. Op. cit., p. 189.

6. Médico epidemiólogo y Máster en Desarrollo Humano, donde el Índice de Desarrollo Humano Territorial de la capital cubana fue su Trabajo de Fin de Máster con la Universidad Católica de Murcia, San Antonio, en 2013.

Feminismo/s 25, junio 2015, pp. 55-70 
Plaza de la Revolución ocupó el tercer puesto. En el espacio marginal que comparten El Fanguito y Las Canteras se presenta el bullying que ocurre en las escuelas primarias y secundarias desde una transversalización de género, ya que estos roles verifican circunstancias especiales ante el acoso escolar.

\section{Bullying e imaginario social}

Ante agresiones físicas, psicológicas o verbales comprobamos que se está generando bullying a partir de un agresor a su víctima, siendo así que en la mayoría de los casos que leemos y escuchamos en las noticias encontramos historias altamente personalizadas, pero ¿qué hay del bullying social cuando tiene lugar entre dos grupos de un mismo espacio urbano? En estos casos ocurre un énfasis en la exclusión o aislamiento que acontece en los actos de la vida cotidiana, en el imaginario de un barrio y su relación siempre colectiva con las mentalidades.

Cuando aparece la marginación de la no existencia también está ocurriendo el bullying, ya que se acosa socialmente a un grupo apartándolo de las actividades en las que normalmente debería participar como miembro de un espacio social definido como lo es la ciudad. Ignorar es atacar la autoestima, cuando los niños y los padres que viven en el barrio Las Canteras rechazan la matrícula de la escuela primaria o secundaria porque a ella asisten los niños y adolescentes de $\mathrm{El} \mathrm{Fanguito.} \mathrm{La} \mathrm{causa} \mathrm{es} \mathrm{el} \mathrm{río} \mathrm{Almendares} \mathrm{contaminado} \mathrm{que} \mathrm{se}$ encuentra del lado de El Fanguito, que termina de hacer insalubre un barrio improvisado en casas de madera, con cubiertas de madera y papel de techo y pisos de morteros ${ }^{7}$; mientras que Las Canteras posee edificios y casas de mampostería, aceras y calles bien delimitadas.

Cuando Pierre Bourdieu ${ }^{8}$ explica la violencia simbólica, subraya la complicidad de las víctimas, puesto que al repetirse cada día, las asimetrías sociales a través de acciones cotidianas se presentan como imposibles de vencer en las mentalidades de los dominados. La búsqueda de otras escuelas por los padres de Las Canteras, así como de opciones de ocio fuera del barrio, están impulsadas por un nivel económico superior que encuentra en la diferenciación la respuesta a la separación que aspira a imprimir en sus niños y adolescentes.

7. Según datos del 2008 del Instituto de Planificación Física y de su dirección provincial de Ciudad de La Habana y municipal de Plaza de la Revolución, así como del Consejo Popular Carmelo y del Parque Metropolitano.

8. GALlegos, María del Carmen. «Violencia escolar y su vínculo con la violencia de género». Revista Iberoamericana sobre niñez y juventud (2012), pp. 33-38. <http://revistarayuela.ednica.org.mx/sites/default/files/Mar\%C3\%ADa\%20del\%20Carmen\%20Gallegos\%20Arg\%C3\%BCello.pdf> consultado el 15-03-2015. 
En El Fanguito las mentalidades no buscan escapar de la prisión marginal, «La educación de los padres es a través de la violencia verbal, se proyectan con una mentalidad falta de comprensión y comunicación $»^{9}$; lo cual lleva a que los niños y adolescentes también practiquen la violencia verbal entre ellos y estén dispuestos a la agresión física. Tampoco falta la violencia física entre los adultos, «a veces los niños participan con ellos» ${ }^{10}$, el alcoholismo, la prostitución, la pobreza creativa, van constituyendo un mundo cerrado donde confinan a la próxima generación.

Lo que está ocurriendo en El Fanguito lo articula Ángela Ferriol en el concepto de marginalidad con la imposibilidad de obtener una vía de acceso al futuro,

La marginalidad es un proceso, no se trata de un estado o de una situación (...) se debe enfatizar en la condición de falta de acceso, por parte de determinados grupos económicos, a la creación de la riqueza y a su disfrute. (...) Muchas veces la marginalidad se expresa en la ausencia o escasez de lo que se ha dado en llamar capital social, es decir, falta de calificación, de cultura. Por supuesto, igualmente se relaciona con la falta de vínculos productivos, son grupos que no tienen tierras, acceso a créditos, ni siquiera a viviendas, y por tanto no tienen una vía que les permita acceder al capital social ${ }^{11}$.

Por la carencia de acceso la marginalidad puede situarse junto a los procesos de pobreza y exclusión social en una interrelación de resultados negativos para la sociedad,

De alguna manera, la exclusión social no es sino el espejo invertido y puede que deformado de la sociedad en que se crea, el subproducto poco presentable que queda como resultado de procesos sociales más amplios y complejos que afectan a los sistemas de producción y de reproducción social. Las transformaciones en la estructura ocupacional, en el mercado de trabajo, en las condiciones laborales y de explotación de los trabajadores, así como todo cuanto afecta a la estructuración de las relaciones familiares y de vinculación social, $(\ldots)^{12}$

En el contexto de estas relaciones significantes de poder entre los dos barrios, los roles de género ocupan su lugar, puesto que no son el único campo por medio del que se articula el poder sino el primario, siguiendo a Pierre Bourdieu, que refiere que los conceptos de género estructuran la percepción y la

9. Entrevista, septiembre de 2012.

10. Entrevista, septiembre de 2012.

11. FERRIOL, Ángela. «¿Entendemos la marginalidad?». Temas 27 (2001), pp.71-72.

12. La exclusión social en España. Un espacio disperso y diverso en intensa transformación. Madrid, Fundación FOESSA, 2008, p. 175. 
organización, concreta y simbólica, de toda la vida social ${ }^{13}$. En una mirada superficial no se perciben grandes diferencias:

«juegan los mismos juegos, aunque las hembras están menos en el río» ${ }^{14}$.

«Ambos van para la calle desde que caminan para aprender a valerse por sí mismo, para su supervivencia según los padres» ${ }^{15}$;

Pero a las mujeres se les exige ir conociendo cómo realizar las labores del hogar, así como más estudio y mejores notas:

«la hembra tiene que tener una carrera; el varón que saque piedra» ${ }^{16}$.

Aparece muy clara la identidad subjetiva que tendrá la mujer en El Fanguito a partir de estos conceptos que norman su vida hacia el cuidado de los niños pequeños y el ocio a través de la televisión, actitudes que le permiten ser aceptadas del lado de Las Canteras, mientras los varones son rechazados por la libertad que tienen de alejarse del hogar, y en su andar errante por el barrio se les permite un ocio agresivo que consta de subirse en los árboles, tirar piedras y bañarse en el río contaminado. Lo interesante del baño en el río es que también se trata un símbolo culturalmente disponible para el discurso de género ya que su reconocimiento con honestidad está transversalizado, pues el padre lo acepta como un hecho que sucede con o sin su permiso, pero las madres y abuelas lo niegan todas.

El significado de estas complejas conexiones también debe entenderse desde la oposición binaria, la cual provee experiencias diferentes para varón y mujer. El gasto que provee la diferenciación en el barrio Las Canteras se hace sensiblemente más profundo en las niñas y adolescentes, puesto que es la madre la que subraya con insistencia que deben realizar cursos y paseos en $\mathrm{El}$ Vedado, y no niega que esa otra realidad debe apartar a las hijas de la confraternidad con las niñas y adolescentes de El Fanguito ${ }^{17}$. Cuestionar estos aspectos de la vida cotidiana es lo que Joan Scott denomina como amenazas a la totalidad del sistema ${ }^{18}$, imprimir un cambio viene por modificar estas pequeñas relaciones de poder, donde abrir un diálogo Fanguito-Canteras

13. SCOTT, Joan. «El género una categoría útil para el análisis histórico». En Martha Lamas (comp.), El género. La construcción cultural de la diferencia sexual. México D.F., PUEG, 2000 , p. 26.

14. Entrevista, septiembre de 2012.

15. Entrevista, septiembre de 2012.

16. Entrevista, septiembre de 2012.

17. Entrevista, diciembre de 2012.

18. SCOTT, Joan. Op. cit., p. 4. 
sobre la educación y las diferencias de la educación ante una hija o un hijo, llevará inevitablemente a encontrar caminos que supriman el bullying social.

Este bullying no permite que se observen entre ambos los puntos empáticos que pueden existir, la supresión del contacto lleva a conformar estereotipos; en este sentido el ser humano deja de ser una víctima de carne y hueso para convertirse en un conjunto de características que se pretende mantener excluidas ${ }^{19}$. Situación que impide a quienes la sufren ejercer sus derechos sociales, las coloca por debajo en una infraclase o underclase, palabras utilizadas por los teóricos norteamericanos, «(...) se ha caracterizado la underclas como una segregación urbanística en guetos, de grupos sociales marginales, sobre todo raciales, y donde existe una elevada concentración de personas y de hogares desestructurados, así como de conductas delictivas ${ }^{20}$.

Al sostener la mujer de El Fanguito el peso de la sujeción en función del varón, las niñas y adolescentes aprenden desde muy pequeñas a actuar sin proyecto de vida propio, aceptan su supeditación como un juego de roles natural que han observado en sus madres, no buscan ser ejes de transformaciones históricas. «De esta manera, toda la historia pasada ha sido obra del varón sin incidencia de la mujer $»^{21}$. En este aspecto negativo encuentra el bullying social una puerta para quebrar las posiciones de marginación, donde las pocas niñas y adolescentes que son aceptadas en Las Canteras ofrecen siempre su ayuda para cuidar niños más pequeños, para limpiarlos o darles de comer. Sin embargo, las mujeres y las niñas tienen experiencias, sentimientos y aspiraciones que pueden compartir más allá de lo doméstico, no es imposible que las mujeres puedan reaccionar y hacer aportaciones creativas al proceso de entendimiento entre los dos barrios.

En este contexto de poca y difícil interacción Fanguito-Canteras, ocurren en el medio escolar dos situaciones negativas: existe una sola escuela para ambos y Las Canteras, que está en posición superior socioeconómica, también lo está en posición numérica. Las acciones de bullying que los niños y adolescentes realizan por imitación de sus padres quedan sin respuesta a causa de que la educación nacional no posee espacios que alcancen a los casos

19. DíAz-Aguado, María José. «Sexismo, violencia de género y acoso escolar. Propuestas para una prevención integral de la violencia». Revista de Estudios de Juventud 73 (2006), pp. 38-57.

20. HeRnANDEZ, Manuel. «Pobreza y exclusión en las sociedades del conocimiento». En Manuel Hernández Pedreño (comp.), Exclusión social y desigualdad. Murcia, Universidad de Murcia, Servicio de Publicaciones, 2008, pp. 15-58.

21. NASH, Mary. «Nuevas dimensiones en la historia de la mujer». En NASH, Mary (comp.), Presencia y protagonismo. Aspectos de la historia de la mujer. Barcelona, Ediciones del Serbal, 1984, pp. 9-50. 
particulares, la dirección de la institución escolar incentiva el diálogo para que al salir del centro estudien juntos, se conozcan más y compartan otras actividades $^{22}$. Los educadores saben que el bullying no puede eliminarse por decreto y buscan en el intercambio cotidiano positivo destruir los ambientes disparadores de violencia ${ }^{23}$, equiparando las relaciones de poder y manejando con cuidado los distintos aspectos de la relación Fanguito-Canteras.

\section{Diseño metodológico aplicado en el barrio El Fanguito}

La investigación-acción-participación también conocida por sus siglas, IAP, es una metodología para borrar barreras, o sea, para establecer espacios de inclusión y democracia. Se consideró adecuado para el caso que se presenta de bullying social en las escuelas primarias y secundarias a las que asisten los niños y adolescentes de El Fanguito y Las Canteras, así como para generar una deconstrucción de las barreras de género.

El potencial de la investigación participativa apunta a la producción de conocimiento, articulando de manera crítica los aportes de la ciencia y del saber popular, con el fin de reorientarlos hacia la acción transformadora de la realidad. A través de sus técnicas, la IAP desencadena intercambios constructivos entre investigador y comunidad en los que se abordan conjuntamente todas las etapas del proceso investigativo y de intervención social ${ }^{24}$.

Una intervención pensada desde fuera de El Fanguito no podrá promover un diálogo genuino contra las posturas marginadoras presentadas. Se validó la IAP como herramienta participativa para la ruptura de las barreras de las mentalidades hacia una intervención social donde se encontraran activas ambas partes. Con la promoción de actividades para el empoderamiento de las mujeres y la convivencia intersocial, la promoción humana mejorará las condiciones de socialización entre los dos barrios. La IAP no comprueba teorías sino que comparte en el análisis, el diálogo y la discusión en la que estuvieron presentes los vecinos de El Fanguito y Las Canteras en la búsqueda de soluciones.

22. Entrevista, septiembre de 2012.

23. Mora, Alma Rosa. Violencia y desigualdad de género en el aula. Del contrato sexual al contrato escolar. Departamento de Sociología rural, Universidad Autónoma de Chapingo, Ciudad de México, p. 40.

http://tumbi.crefal.edu.mx/decisio/index.php?option=com_content\&view=article\&id $=106 \&$ Itemid $=147>$ consultado el 19-05-2015

24. CONTRERAS, Rodrigo. «La Investigación Acción Participativa (IAP): revisando sus metodologías y sus potencialidades». En John Durston, Francisca Miranda (comps.), Experiencias y metodologías de la investigación participativa. Santiago de Chile, CEPAL, 2002, pp. 9-18. 
Con el propósito de limitar la posición de observador externo del investigador, se buscaron las potencialidades de la propia comunidad de El Fanguito, los saberes que emplean en su propio desarrollo. Esto resultó en el encuentro con el Grupo Vida que consta de un equipo de jóvenes que realizan un programa de animación sociocultural empírico con el barrio El Fanguito, a partir de su fe cristiana. Cada uno es miembro de la Capilla Jesús Obrero que se encuentra en los límites entre El Fanguito y Las Canteras, donde las hermanas dominicas misioneras de la Sagrada Familia reciben a todos sin distinción; algunos son vecinos de dentro de El Fanguito, otros de Las Canteras y otros de El Vedado. Sus reuniones de crecimiento espiritual les llevaron hacia un compromiso para con los niños y adolescentes de El Fanguito, para conducirles con cuentos, representaciones, manualidades y juegos deportivos, al incremento de los valores y el amor a la naturaleza, sin tener idea de que conducen un programa de animación sociocultural empírico.

El investigador solo dinamizó y orientó sus actividades, el compromiso realizado se movió de la investigación al grupo y no en el sentido contrario de una asimilación acrítica por parte del Grupo. En ellos existe un alto sentido de conciencia aunque desconocían la importancia de sus actos en sistematicidad y voluntad que se había extendido por El Fanguito en más de cinco años. Es un Grupo que aprende enseñando y tiene muy claros los obstáculos que impiden romper la marginación social, no conocían el significado de bullying o de empoderamiento de las mujeres pero reconocían su significado en la vida cotidiana del barrio.

En su aspecto psicosocial, la IAP hace énfasis en la autodeterminación de los destinatarios y en la búsqueda de decisiones desde dentro sobre los problemas que los aquejan. El ser protagonista histórico es una necesidad humana, como bien lo describió el pedagogo Paulo Freire cuando enfoca la explicación del mundo a partir del contexto donde se encuentra el ser humano que puede ser susceptible de educación aún en la edad adulta ${ }^{25}$.

Entre los valores que han guiado la intervención psicosocial está el rol de sujeto activo que se asigna a los participantes de la comunidad, al mismo tiempo que constituyen objetos de la intervención. El proceso de intervención supone el desarrollo de una relación de interacción e influencia entre el equipo interventor y los destinatarios, de tal manera que, como resultado del

25. Freire, Paulo y Betto, Frei. Esa escuela llamada vida. Buenos Aires, Editorial LEGASA S.A., 1988 , p. 30. 
proceso de colaboración mutua, cada parte modifique sus visiones iniciales de la situación ${ }^{26}$.

\section{La técnica utilizada en la investigación-acción-participación (IAP)}

Se utilizó la técnica DAFO (Debilidades, Amenazas, Fortalezas y Oportunidades $)^{27}$ para autodiagnóstico local a través del Grupo Vida, respetando la investigación como ese proceso reflexivo que lleva luego a la acción, pero esta se nutre a su vez de más conocimiento para investigar, al tiempo que se considera siempre a las comunidades de El Fanguito y Las Canteras como sujetos activos en su transformación.

Asimismo, se subrayó la necesidad de crear competencias psicosociales ya que esto, «(...) implica el ejercicio de acciones eficaces en la interacción con el medio (y consigo mismo), las cuales se realizan sobre la base de los propios recursos y de los recursos externos, adecuadamente percibidos y evaluados ${ }^{28}$.

\section{La Técnica DAFO, (Debilidades, Amenazas, Fortalezas y Oportunidades)}

El Grupo Vida incluyó la técnica DAFO como parte de sus estrategias educativas en el barrio El Fanguito, con el objetivo de incluir en sus acciones la creación de un diálogo Fanguito-Canteras que elimine el bullying social y haga crecer el empoderamiento de las mujeres. Al ser un grupo heterogéneo, sus objetivos de trabajo con los niños y adolescentes del barrio son múltiples, les interesa la superación de las deficiencias en la concentración y el análisis que se muestran a través de la pobreza del lenguaje. También enfocan sus actividades hacia el aprovechamiento escolar y la legitimidad del conocimiento para aminorar las ausencias a clases y repasos, junto a la despreocupación y violencia de los padres ante bajas calificaciones. Asimismo se ocupan de la salud para la disminución del baño en el río contaminado junto a la caza y pesca y su sustitución por el ocio sano ${ }^{29}$.

Estos jóvenes proceden de los tres espacios: El Fanguito, Las Canteras y El Vedado, donde sus puntos de vista sobre las necesidades del barrio se hacen

26. KRAUSE, Mariane. «Investigación-Acción-Participativa: una metodología para el desarrollo de autoayuda, participación y empoderamiento». En John Durston y Francisca Miranda (comps.). Op. cit., p. 42.

27. BASAGOITI, Manuel; BRU, Paloma y LoREnZANA Concha. Investigación-acción-participativa de bolsillo. Madrid, Acsur-Las Segovias, 2001, p. 23. <www.acsur.org/IMG/pdf/ Guia_IAP.pdf>, consultado el 19-03-2015.

28. Mariane Krause. Op. cit., p. 44.

29. El Grupo Vida no solo provee actividades de ocio sano, también limpia y chapea espacios baldíos con la ayuda de los padres para sensibilizarlos con la necesidad de una diversión sin prejuicio. 
múltiples en correspondencia con sus profesiones: el derecho, la medicina, el cine, la economía o la pintura. Sin embargo, existe homogeneidad entre ellos, sus edades oscilan entre 20 y 30 años, casi todos profesionales o estudiantes universitarios, predomina la soltería, aunque los dirige una joven casada, cuyo esposo también participa del Grupo, y con respecto al género se encuentran balanceados.

La DAFO proveyó datos muy valiosos para la continuidad del trabajo:

En el análisis interno, las Debilidades fueron: La soledad en el sentido familiar les produce tristeza y también comportamientos nocivos como el baño en el río y la violencia, la cual muestran siempre estando a la defensiva y subrayando en sus juegos las posiciones de poder. Esta soledad también implica que queman etapas, pues están criados fuera, en los espacios externos del barrio, en la sobrevivencia que les obliga a madurar. En la vida cotidiana los padres pasan un mínimo de tiempo con ellos y los educan a partir de amenazas de violencia física, -te voy a..., si no haces...- Entonces se educan con poca creatividad e imaginación, no hay en los hogares una búsqueda del desarrollo de capacidades y habilidades.

La comunicación entre los propios niños y adolescentes es pobre, muchos son introvertidos y otros no saben expresar lo que sienten, no establecen un ambiente de confianza porque se burlan demasiado entre ellos, sobre todo a los que no cumplen con el estereotipo del barrio de ser achispado y fuerte, y de no permitir que nadie haga su voluntad contigo. También la introversión viene por los problemas en los núcleos familiares, las historias ocultas, la violencia, la droga, la prostitución que saben no pueden contar, y aprenden excusas y mentiras según sea el caso. Son hiperactivos, tienen energía y deseos de hacer, pero sin analizar, sin profundizar en sus ideas y actitudes, lo que los lleva a estar, muchas veces, desorientados, tener poca concentración y no plantearse expectativas y motivaciones, algunos ni se plantean qué hacer en el futuro, porque se les enseña mucho a vivir en el presente, en lo que se consigue día a día para vivir.

Mientras en ese mismo aspecto las Fortalezas estuvieron en: desde que aprenden a caminar son muy independientes por esa libertad que tienen de vagar por el barrio, son fuertes y saben defenderse, nadie del exterior puede venir a dañarlos, o a llevárselos. Anhelan dar cariño, gustan mucho de esa interacción de dar y recibir, son muy receptivos para devolver cariño a quien se lo da, muestran sensibilidad y buenos sentimientos. Para ellos la familia es una unidad muy fuerte, aprenden desde temprano a compartir lo poco que tienen entre todos. No saben fingir, ni siquiera por educación, dicen lo que piensan. Se identifican entre ellos por ser del barrio, son un bloque frente 
a otros grupos, el medio los une, lo desarrollan casi por instinto, hay una mentalidad de sentirse unidos frente a otro $u$ otros que no sean del barrio. Se aprecia respeto hacia el Grupo Vida, por las enseñanzas y valores que con ellos aprenden.

En el análisis externo las Amenazas estuvieron en: la violencia del barrio desde el punto de vista físico o de las conductas hace que el medio social sea áspero, difícil, muchos ejemplos negativos de padres y vecinos. Hay presencia de drogas, prostitución, gente que entra y sale de la cárcel, malas palabras y mucha violencia verbal dentro de la familia. El bajo nivel cultural no es siempre educativo, pero sí mental, aquellos que estudiaron y los que no lo hicieron prefieren el dinero rápido. Los maestros gritan demasiado, no tienen un trato suave y agradable, dan conocimientos mínimos y con eso creen que han cumplido con su deber.

Esta vida cotidiana puede impedir que los niños y adolescentes tengan acceso a otro futuro, a conocer otras realidades y a escoger otros comportamientos. A lo que se agrega la falta de higiene y confort del barrio que lo hace un medio hostil y peligrosamente contaminado, donde tampoco se puede hablar de un calor del hogar. Frente a este telón de fondo aparece la falta de espacios para la diversión, los padres casi exclusivamente salen con los hijos del barrio para turnos médicos, es con el Grupo Vida con quien pasean y hacen actividades fuera sobre todo en verano durante las vacaciones. El río es la fuente de diversión para los tiempos de ocio, el baño, la caza, la pesca, siendo los padres los que los dejan vagar solos por un pequeño barrio con su río contaminado.

Asimismo las Oportunidades estuvieron en: aunque la hiperactividad les impide el análisis, este es un camino seguro para el aprendizaje ya que siempre están dispuestos para la actividad física y con ella se les puede llevar a la reflexión. Los padres tienen una profunda confianza en el Grupo Vida no solo para las actividades que prepara en el barrio a la vista de todos, también para los paseos o actividades que duren varios días separados de la casa. También el barrio muestra su lado positivo, los vecinos son solidarios, se ayudan y se avisan para comprar la canasta básica, hay mucha preocupación por que nadie se quede sin comer. No olvidan los padres que sus hijos necesitan de su sensibilidad, algunos han apoyado las actividades del Grupo ocasionalmente para higienizar el barrio y que los niños y adolescentes puedan jugar en un mejor ambiente.

La animación sociocultural del Grupo Vida en El Fanguito puede implicar un desafío en busca de la combinación económica, social, cultural y ecológica. Al focalizar un trabajo que integre a padres e hijos, ganará un espacio que no 
solo permitirá un crecimiento de la animación sociocultural en calidad y cantidad, sino el paso hacia el diálogo Fanguito-Canteras. En el trabajo con los niños y adolescentes, el Grupo ha comprobado en la práctica que existen personas con otras experiencias vivenciales que difieren de muchas encontradas en el barrio El Fanguito, y estas personas están en disposición de conducirles por un camino de acceso al futuro. Siendo así, el barrio puede tener esperanza de futuro para sus hijos, no solamente por todo el esfuerzo del Grupo Vida sino porque se han sensibilizado personalidades de la cultura cubana como la cantautora, y embajadora de Buena Voluntad del Fondo de las Naciones Unidas para la Infancia, UNICEF ${ }^{30}$, Liuba María Hevia. Otro aspecto alentador es que a nivel nacional existen actores sociales comprometidos con el desarrollo humano y la sostenibilidad, quienes deberán ser contactados para que avance el proyecto socio-cultural en un espacio físico determinado, logro que deberá gestionarse con el Ministerio de Ciencia Tecnología y Medio Ambiente. El Programa de las Naciones Unidas para el Desarrollo (PNUD) en Cuba trabaja desde la perspectiva del desarrollo humano local, se ocupa de la calidad de vida y el desarrollo humano en municipios seleccionados, llevando adelante el fortalecimiento del gobierno local, la participación comunitaria, las perspectivas de género y el uso de las tecnologías. En resumen, el proyecto que lleva adelante el Grupo Vida y sus retos es en cuestión viable, pero también necesario, es un sueño, pero también una realidad en proceso. Proveer para el futuro de estos niños y adolescentes es hacerlo para los de todo el país, contribuyendo a que el desarrollo humano sostenible sea esa realidad que empodera ciudadanos y elimina las conductas nocivas para la esencia del ser, en Cuba.

\section{Conclusiones}

Cuba es un escenario de pobreza multidimensional con niveles de amparo estatal de calidad óptima que necesita focalizar en lugares marginales atenciones que no requieren su universalización en todo el país. En estas zonas, como la que ocupan El Fanguito y Las Canteras, se hace presente el acoso escolar con amplios matices de género. El acoso es aprendido por los hijos directamente de sus padres, se confirma a través de la vida cotidiana negando la presencia del otro. El barrio El Fanguito acepta ser ese otro y se encierra con sus hijos en un mundo sin salida; solo las normas que rigen la vida de la mujer, con su higiene y mayor fuerza y pasividad, permiten el contacto con Las Canteras. Al varón se le exigen en El Fanguito posiciones de mayor fuerza

30. UNICEF, El Estado Mundial de la Infancia, 2012. En: http://www.unicef.org/spanish/ sowc2012/, consultado el 03-03-2014.

Feminismo/s 25, junio 2015, pp. 55-70 
y agresividad, no tiene escapatoria, con lo cual las víctimas del acoso social se autorreproducen.

Cuando a través de la construcción de género tiene lugar el contacto Fanguito-Canteras, estos espacios pueden utilizar las posiciones de ayuda doméstica como un canal para compartir proyectos de vida y conectar los dos barrios, en un diálogo que elimine el bullying principalmente en la escuela. El apoyo para este paso, tanto de la propia escuela como desde el Grupo Vida, aún deberán ganar mayor espacio, sobre todo hacia la educación de adultos, o sea, de los padres; las normativas de la vida cotidiana tendrán que abrirse hacia la equidad de género o el aprovechamiento de un diálogo FanguitoCanteras no será total. Trabajar en estos retos abrirá un camino y una experiencia beneficiosa para otros niños y adolescentes cubanos en situaciones de marginalidad.

\section{Referencias bibliográficas}

BASAgoiti, Manuel; Bru, Paloma y Lorenzana, Concha. Investigación-acciónparticipativa de bolsillo. Madrid, Acsur-Las Segovias, 2001, pp. 17-29. <www. acsur.org/IMG/pdf/Guia_IAP.pdf>, consultado el 19-03-2015.

CARREÑO, Miryam. "Teoría y práctica de una educación liberadora: el pensamiento pedagógico de Paulo Freire». Cuestiones Pedagógicas 20 (2009/2010), pp. 195-214.

CONTRERAS, Rodrigo. «La Investigación Acción Participativa (IAP): revisando sus metodologías y sus potencialidades». En John Durston, Francisca Miranda (comps.), Experiencias y metodologías de la investigación participativa. Santiago de Chile, CEPAL, 2002, pp. 9-17.

Couceiro, Avelino Víctor. Historia de Plaza de la Revolución. La Habana, Publicitaria Imágenes del CIMEX, 2003.

CROSAS, Carles. Retículas verdes, nuevas ciudades decimonónicas. El paradigma del vedado, "ensanche jardín» de la habana. Barcelona, Universidad de Catalunya, 2012, pp. 27-40.

$<$ http://www.revistaiberoamericanadeurbanismo.pdf>, consultado el 1-10-2012.

Díaz-Aguado, María José. «Sexismo, violencia de género y acoso escolar. Propuestas para una prevención integral de la violencia». Revista de Estudios de Juventud 73 (2006), pp. 38-57.

ESPINA, Mayra Paula. «El caso cubano en diálogo de contraste», en Políticas de atención a la pobreza y la desigualdad. Examinando el rol del estado en la experiencia cubana. Buenos Aires, Consejo Latinoamericano de Ciencias Sociales (CLACSO), 2008, pp. 95-192.

FERRIOL, Ángela. «¿Entendemos la marginalidad?». Temas 27 (2001), pp. 60-75. 
FERRIOL, Ángela. Políticas Sociales y reformas estructurales: Cuba a principios del siglo XXI. Comisión Económica para América Latina y el Caribe (CEPAL), Naciones Unidas, Programa de las Naciones Unidas para el Desarrollo (PNUD), Instituto Nacional de Investigaciones Económicas (INIE), 2004.

FOESSA. La exclusión social en España. Un espacio disperso y diverso en intensa transformación. Madrid, Fundación FOESSA, 2008, pp. 169-177.

Freire, Paulo y Betto, Frei. Esa escuela llamada vida. Buenos Aires, Editorial LEGASA S.A., 1988.

GALLEGOS, María del Carmen. «Violencia escolar y su vínculo con la violencia de género». Revista Iberoamericana sobre niñez y juventud. (2012), pp. 33-38. <http://revistarayuela.ednica.org.mx/sites/default/files/Mar\%C3\%ADa\%20del \%20Carmen\%20Gallegos\%20Arg\%C3\%BCello.pdf> consultado el 15-03-2015.

Hernandez-Lorenzo, Maité y VAliño, Omar. Vicente Revuelta Monólogo. La Habana, Ediciones Alarcos, 2011.

HeRnANDEZ PeDReÑo, Manuel. «Pobreza y exclusión en las sociedades del conocimiento». En Manuel Hernández Pedreño (comp.), Exclusión social y desigualdad. Murcia, Universidad de Murcia, Servicio de Publicaciones, 2008. pp. 15-58.

KRAUSE, Mariane. «Investigación-Acción-Participativa: una metodología para el desarrollo de autoayuda, participación y empoderamiento». En John Durston, Francisca Miranda (comps.), Experiencias y metodologías de la investigación participativa. Santiago de Chile, CEPAL (2002), pp. 41-56.

Mora, Alma Rosa. «Violencia y desigualdad de género en el aula. Del contrato sexual al contrato escolar». Decisio 2010, pp. 37-41. http://tumbi.crefal.edu. $\mathrm{mx} /$ decisio/index.php? option=com_content $\& v i e \mathrm{w}=$ article $\&$ id $=106 \&$ Ite mid $=147>$ consultado el 19-05-2015

NASH, Mary. «Nuevas dimensiones en la historia de la mujer». En Nash, Mary (comp.), Presencia y protagonismo. Aspectos de la historia de la mujer. Barcelona, Ediciones del Serbal, 1984, pp. 10-37.

SCOTT, Joan. «El género una categoría útil para el análisis histórico». En Martha Lamas (comp.), El género. La construcción cultural de la diferencia sexual. México D.F., PUEG, 2000, pp. 2-31.

UNICEF, El Estado Mundial de la Infancia, 2012. $<$ http://www.unicef.org/spanish/sowc2012/>, consultado el 03-03-2014.

Feminismo/s 25, junio 2015, pp. 55-70 